Supporting Information 


\section{Chemically Functionalized Silk for Human Bone Marrow-Derived Mesenchymal Stem Cells Proliferation and Differentiation}

Ke Zheng ${ }^{1,2}$, Ying Chen ${ }^{2}$, Wenwen Huang ${ }^{2}$, Yinan Lin ${ }^{2}$, David L. Kaplan ${ }^{*, 2}$ and Yimin Fan $^{*, 1}$

1 Jiangsu Key Lab of Biomass-based Green Fuel \& Chemicals, College of Chemical Engineering, Nanjing Forestry University, Nanjing 210037, China

2 Department of Biomedical Engineering, 4 Colby Street, Tufts University, Medford, MA 02155, United States

\section{AUTHOR INFORMATION}

Corresponding author: Prof. Yimin Fan: fanyimin@njfu.edu.cn; Prof. David L.

Kaplan: david.kaplan@tufts.com

\section{ASSOCIATED CONTENT}

\section{Supporting Information}

Theoretical maximum concentration of oxidized carboxyl group in SF protein

Figure S1 TGA curves of M-OxSF and M-SF scaffolds

Figure S2 Mechanical properties of dry state scaffolds 
Figure S3. Representative stress-strain curves of scaffolds in PBS bath

Figure S4, S5 XPS and XRD spectra of the surface of mineralized scaffolds

Figure S6, S7 Cell viability and morphological analysis of hMSCs on scaffolds

Table S1 Q-PCR Primer list

\section{The Theoretical Maximum Concentration of Oxidized Carboxyl Group in SF}

\section{Protein}

The theoretical maximum concentration of oxidized carboxyl group in SF protein was calculated by the molecular weight (heavy chain, 391,593 Da) and the content of serine in SF molecular chain $(12.1 \%, 635$ residue). The formula was:

$$
\mathrm{C}_{(\mathrm{COOH})}=\frac{1}{391.593} \times 635 \times 10^{-3}=1.62 \mathrm{mmol} / \mathrm{g} \text { protein }
$$

Schemes S1. Theoretical maximum concentration of oxidized carboxyl group in SF.

\section{The TGA of M-SF and M-OxSF scaffolds}

TGA of M-SF and M-OxSF scaffolds were performed on a TA Instruments Q500 (TA Instruments, New Castle, DE, USA) thermogravimetric analyzer at a heating rate of $10^{\circ} \mathrm{C} / \mathrm{min}$ from room temperature to $600^{\circ} \mathrm{C}$ in a nitrogen purge gas flow of $50 \mathrm{~mL} / \mathrm{min}$. The initial sample mass was in the range between 4.0 and $5.0 \mathrm{mg}$. 


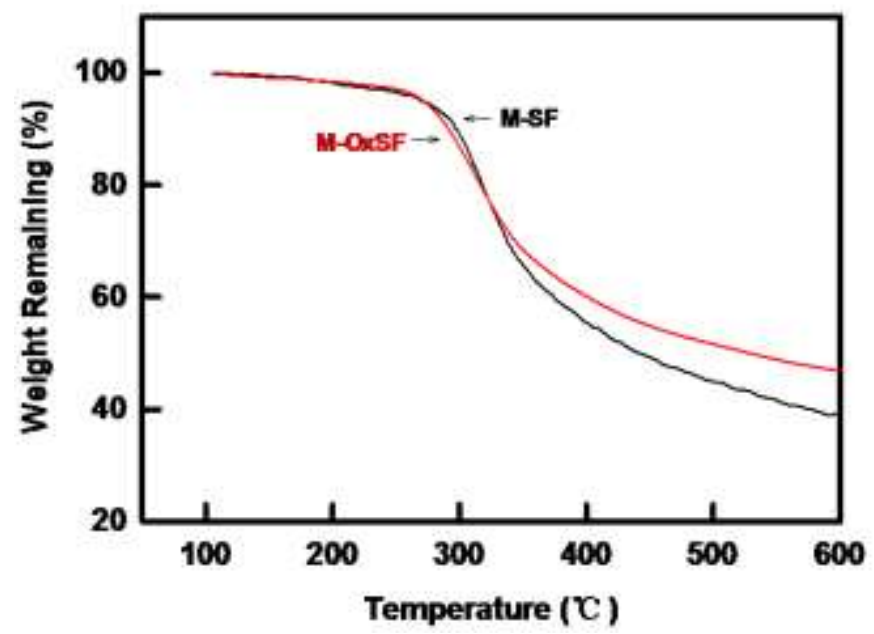

Figure S1. TGA curves of M-OxSF and M-SF scaffolds. The residue of the M-OxSF and M-SF samples were $50.8 \%$ and $44.3 \%$ at $600^{\circ} \mathrm{C}$, respectively.

\section{Mechanical Properties of Dry State SF and OxSF Scaffolds}

Unconfined compressive mechanical testing of the scaffolds was followed by the method described above without PBS bath, and performed on an Instron 3366 testing frame (Instron Corp., USA) equipped with a $0.5 \mathrm{kN}$ load cell.

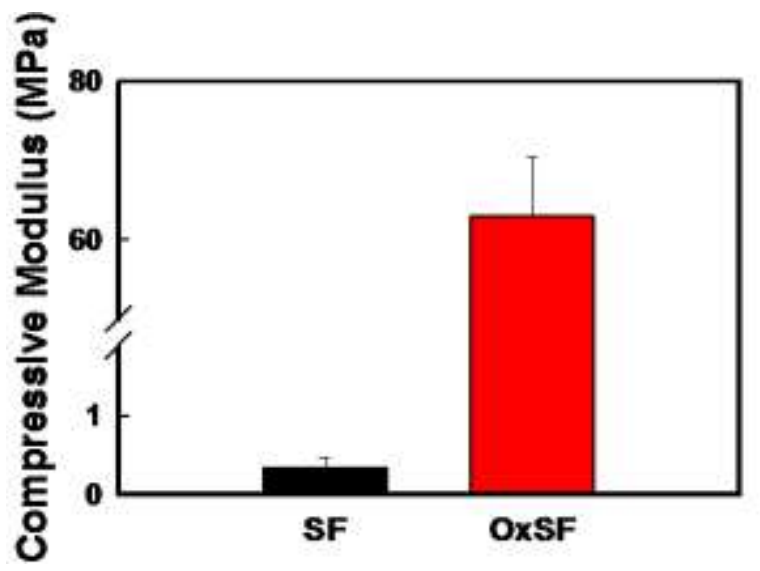

Figure S2. Compressive modulus of SF and OxSF scaffolds in dry state. The OxSF scaffolds obtained a compressive modulus of $53.64 \pm 11.8 \mathrm{MPa}$, at least 100 times higher than the compressive modulus of the SF scaffolds at $0.36 \pm 0.12 \mathrm{MPa}$.

\section{Stress-Strain Curves of SF and OxSF Scaffolds}

Unconfined compressive mechanical testing of SF, OxSF, M-SF and M-OxSF were 
carried out by the method described in part "2.7 Mechanical Properties of Scaffolds".

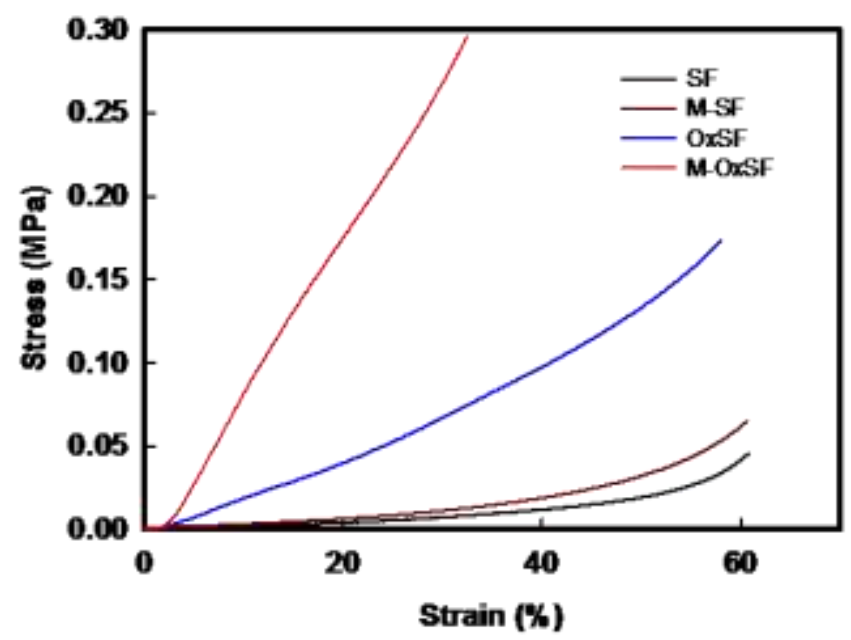

Figure S3. Representative stress-strain curves of SF, OxSF, M-SF and M-OxSF scaffolds.

\section{The Identification of HA on OxSF Scaffolds}

The HA on OxSF scaffolds was analyzed by X-ray photoelectron spectroscopy (XPS) and X-ray diffraction (XRD). The XPS experiments were performed on a K-Alpha XPS system (Thermo Fisher Scientific, MA, USA) using monochromatic soft aluminum K $\alpha$ X-ray sources $(\mathrm{hv}=1361 \mathrm{eV}$ ). Survey scans (spot $400 \mu \mathrm{m}$, pass energy $200 \mathrm{eV}$ ) and elemental scans (spot $400 \mu \mathrm{m}$, pass energy $50 \mathrm{eV}$ ) were performed using a flood gun (charge neutralizer) setting of $5 \mathrm{eV}$ and nickel wire mesh held over the sample to prevent charging of the sample surface. The XRD experiments were performed on an Ultima IV multipurpose X-ray diffraction system (Ultima IV, Rigaku, Japan), employing $\mathrm{Cu} \mathrm{K \alpha}$ source (40 kV and $30 \mathrm{~mA}$ ). 
A

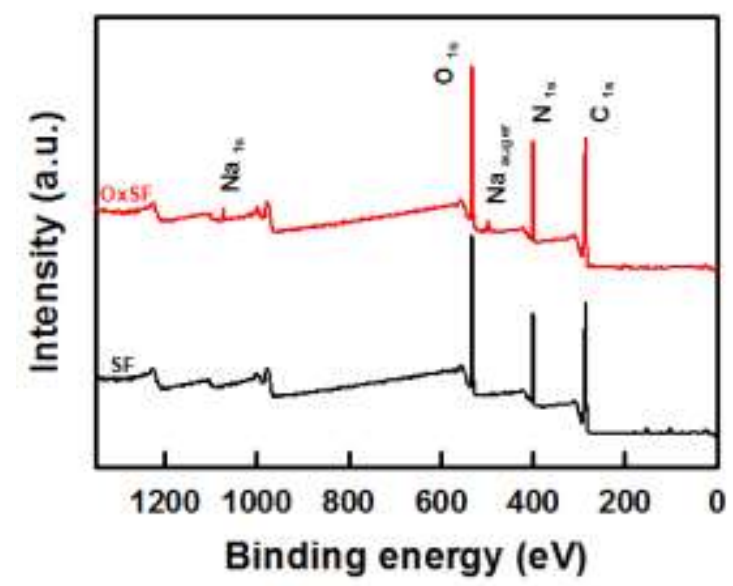

B

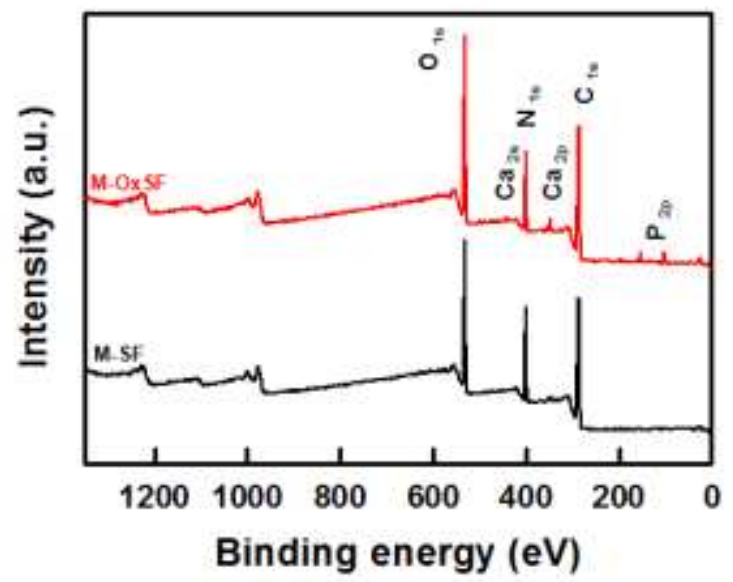

Figure S4. A) XPS spectra of SF and OxSF scaffolds. The peak of Na was detected in OxSF scaffolds due to the sodium carboxylate. B) XPS spectra of M-SF and M-OxSF scaffolds. The peaks of Ca and P were detected in the M-OxSF scaffolds, while these elements were not detected in the M-OxSF scaffolds.

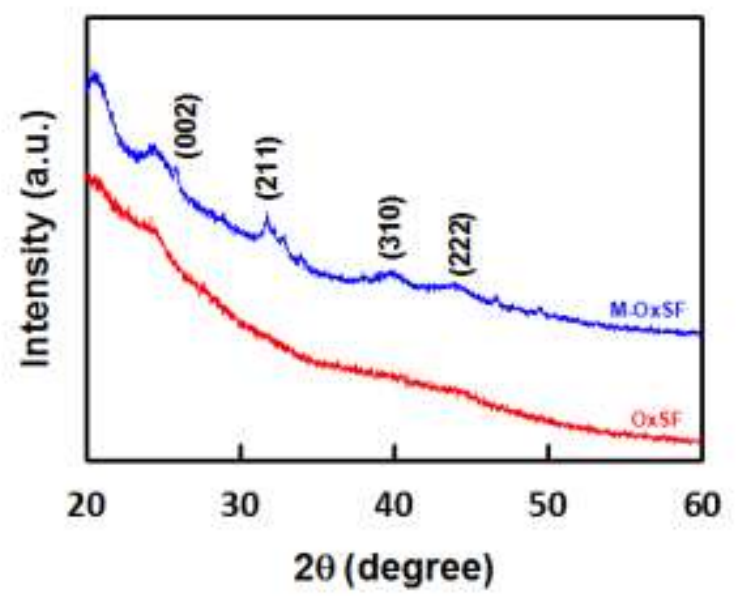

Figure S5. XRD spectra of OxSF and M-OxSF scaffolds. The patterns of M-OxSF revealed that all the peaks are well defined and assigned only to crystalline HA.

\section{Cell Viability Assay}

hMSC metabolic activity was quantified using the alamarBlue ${ }^{\circledR}$ assay (Invitrogen, \#DAL1100) according to the manufacturer's instructions. Briefly, $1 \mathrm{~mL}$ of a solution containing proliferation medium (or differentiation medium) with $10 \%$ alamarBlue ${ }^{\circledR}$ solution was added to 3 wells from each type of silk scaffold, and incubated for 2 hours. A $100 \mu \mathrm{L}$ mixture was then taken from each well, and analyzed for fluorescence 
exciting at $560 \mathrm{~nm}$ and recording the emission at $590 \mathrm{~nm}$. Background fluorescence from the alamarBlue ${ }^{\circledR}$ solution alone was subtracted, and the sample values from 3 wells of each culture were averaged.

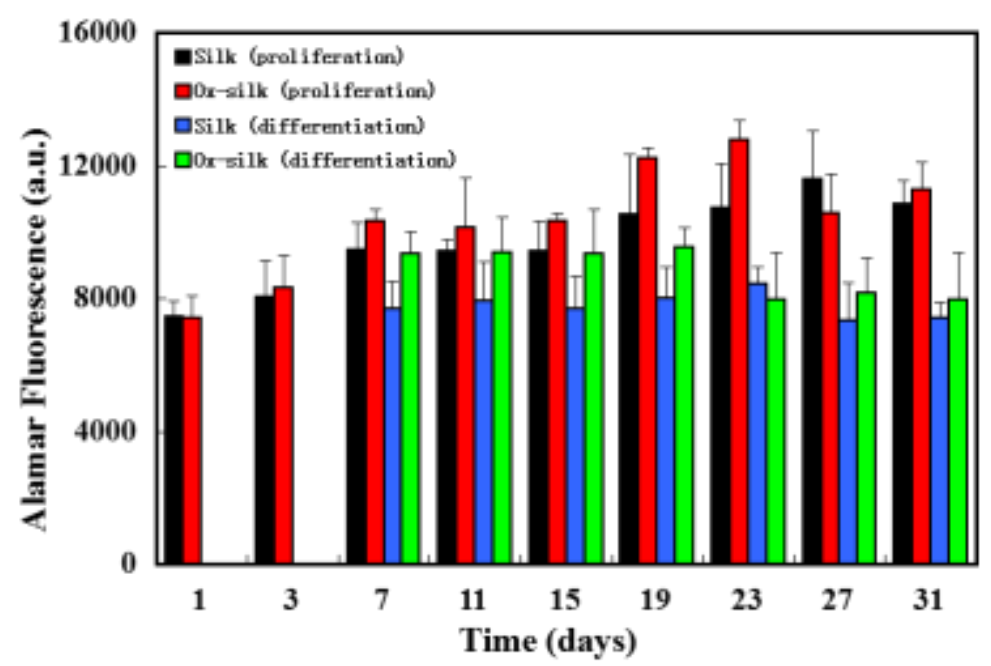

Figure S6. Measure of the viability using the alamarBlue ${ }^{\circledR}$ assay for cells in proliferation and differentiation media on the scaffolds. Half of the samples were changed to differentiation medium after being cultured in proliferation medium for 3 days. Each type of scaffolds showed no cytotoxicity to the proliferation or differentiation of the hMSCs.

\section{Morphological Analysis of hMSCs by Scanning Electron Microscopy}

After 4 weeks culture in osteogenic medium, samples were washed in PBS and then fixed in $2 \%$ glutaraldehyde in sodium cacodylate buffer for $2 \mathrm{~h}$. Constructs were washed in buffer and freeze-dried overnight. The samples were coated with gold and palladium and imaged by SEM (Zeiss UltraPlus SEM, Carl Zeiss SMT Inc., Peabody, MA) at a voltage of 4 to $5 \mathrm{kV}$. 

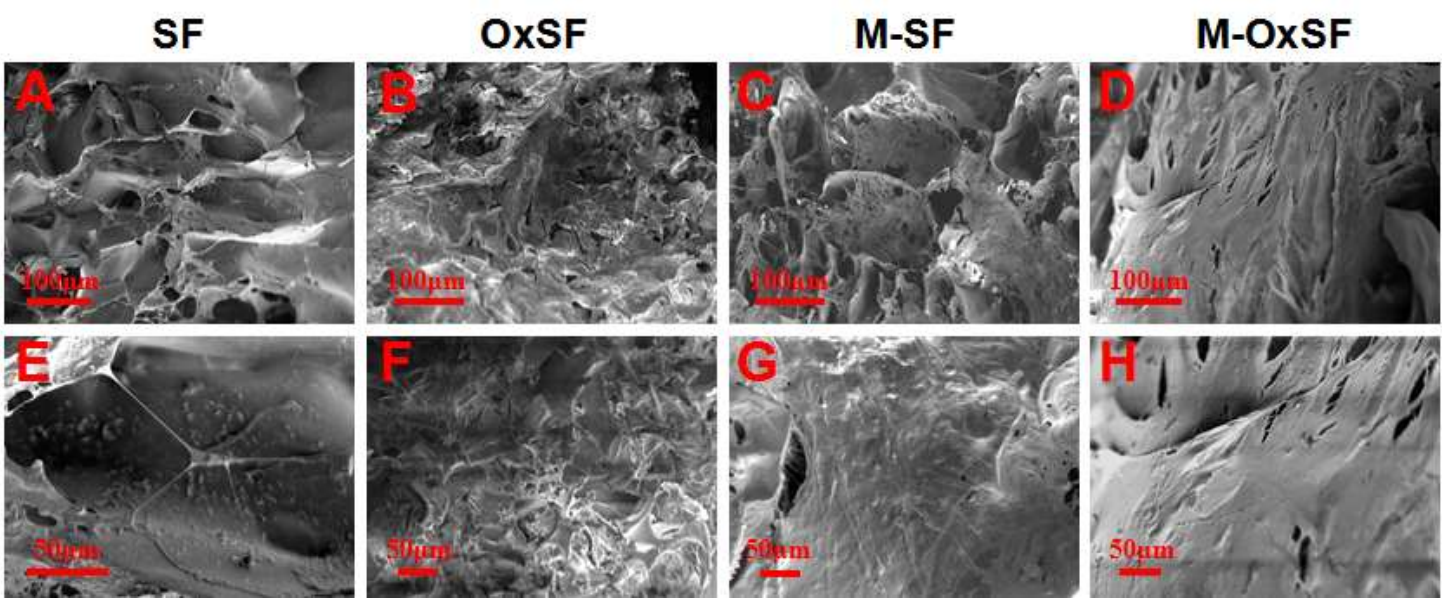

Figure S7. SEM images of scaffolds with seeded of hMSCs (4th week). The bottom images (E-H) are zoomed in images from the top images (A-D). The OxSF scaffolds and scaffolds with mineralization (M-SF and M-OxSF) were covered with extracellular matrix from the hMSCs.

\section{Q-PCR Primer List}

Table S1. Q-PCR primers for GAPDH, ALP, Col1a1, OPN and OC.

\begin{tabular}{lll}
\hline Gene products & Forward & Reverse \\
\hline GAPDH & GAAGGTGAAGGTCGGAGTC & GAAGATGGTGATGGGATTTC \\
ALP & GTGAACCGCAACTGGTACTC & GAGCTGCGTAGCGATGTCC \\
Col1a1 & GAGGGCCAAGACGAAGACATC & CAGATCACGTCATCGCACAAC \\
OPN & GAAGTTTCGCAGACCTGACAT & GTATGCACCATTCAACTCCTCG \\
OC & CACTCCTCGCCCTATTGGC & CCCTCCTGCTTGGACACAAAG \\
\hline
\end{tabular}

